(C) J. Petrek

\title{
PICTORIAL COGNITIVE TASK SOLVING AND DYNAMICS OF EVENT - RELATED DESYNCHRONIZATION
}

\author{
Josef Petrek
}

\author{
Department of Physiology, Faculty of Medicine and Dentistry, Palacky University, Olomouc, Czech Republic \\ e-mail:fesoj@tunw.upol.cz
}

Received: July 22, 2008; Accepted: September 25, 2008

Key words: Cognitive task/Total Power/Frequency at Maximum Power/ERD

Aims: To analyze the event-related desynchronization/synchronization (ERD/ERS) attended with the mental load arising while solving two cognitive tasks. The features of the presented visual stimulus determined the type of cognitive task that should be solved.

Methods: For each experimental task and everybody's subject FFT Analysis module calculated the total power histograms from a fixed frequency band $(3-20 \mathrm{~Hz}$ or $8-13 \mathrm{~Hz}$ ) for each 3-second EEG sample with $50 \%$ overlay and all electrodes. From the histograms the software derived two FFT single values - The average Total Power (TP) and Frequency at Maximum Power (FMP).

Results: It has been shown that during the solution of cognitive tasks the marked changes of ongoing EEG activity appeared. The short lasting and localized amplitude decrease in rhythmic activity (ERD) and the change of EEG frequency were among the most frequent. The ERD extent was determined by an informational content of processed visual stimuli and by the site of scalp-recording electrode. A higher mental load related to the solution of cognitive tasks shifted the average FM to lower frequencies.

Conclusion: The suitability of an analysis of ongoing EEG activity to uncover differences in people's brain activation patterns when engaged in performing cognitively demanding tasks was proved.

\section{INTRODUCTION}

Berger $^{2}$ was the first who proposed the idea that fast changes in brain activity as recorded in human electroencephalogram (EEG) might be related to specific mental activities. He was brought to this conclusion by the observation that EEG of subjects engaging in mental activities such as solving complex mathematical tasks become "desynchronized", i.e. the relatively slow waves of the alpha rhythm predominating during rest are replaced by fast and irregular oscillations at higher frequencies. Since then a number of papers have been published (see e.g. review ${ }^{25}$ ), in which Berger's idea is further developed.

In 1977, Pfurtscheller and Aranibar ${ }^{21}$ described the characteristic change of ongoing EEG (short-lasting decrease/increase in rhythmic activity) that occurs in relation to an event. They designate the change as the "event-related desynchronization/synchronization" or ERD/ERS. Detailed study of ERD, which then followed (see e.g. 6, 8, 11, 12, 17, 22, 23, 24) showed that the ERD represents a good electrophysiological correlate of cortical activation related to stimulus processing. It also gives characteristics of cortical areas preparing to process sensory information or being ready to execute a motor command. Therefore, ERD and ERS are recognized as valuable tools in neurocognitive research. The review of Klimesch ${ }^{6}$ and monographs of Faber ${ }^{3,4}$ and especially papers presented in the book "Event-Related Dynamics of Brain Oscillations", edited by Neuper and Klimesch ${ }^{13}$ in 2006, confirm it unambiguously.
Results of our recent paper ${ }^{14}$ also point at the high suitability of the ERD method to uncover differences in people's brain activation pattern when they are engaged in performing cognitively demanding tasks. The higher mental load pertinent to active searching for identical pictures in a group of similar ones resulted in the prominent ERD localized especially at frontal and parietal recording sites.

Allowing for literature data and our earlier results we decided to analyze ERD/ERS attended with mental load arising during the solving of the two cognitive tasks. The features of presented visual stimulus determined the type of cognitive task that should be solved. The average Total Power, the Frequency at Maximum Power and the dynamics of both in different brain areas was assessed for each type of cognitive tasks. The paper summarizes the main results.

\section{METHODS}

The experimental paradigm, procedures, features of visual stimuli and method of data acquisition were described in detail in our recent paper ${ }^{15}$. Here only the principles of the data analysis are described.

SciWorks version 5 with DataWave CP Analysis Modules and Data Editing Software were used for an offline analysis of experimental data. The analysis ran as follows: A visual elimination of distorted records, sorting records into groups according to the type of mental task, digital filtering of records (bandpass $1-30 \mathrm{~Hz}$ ), and cutting 

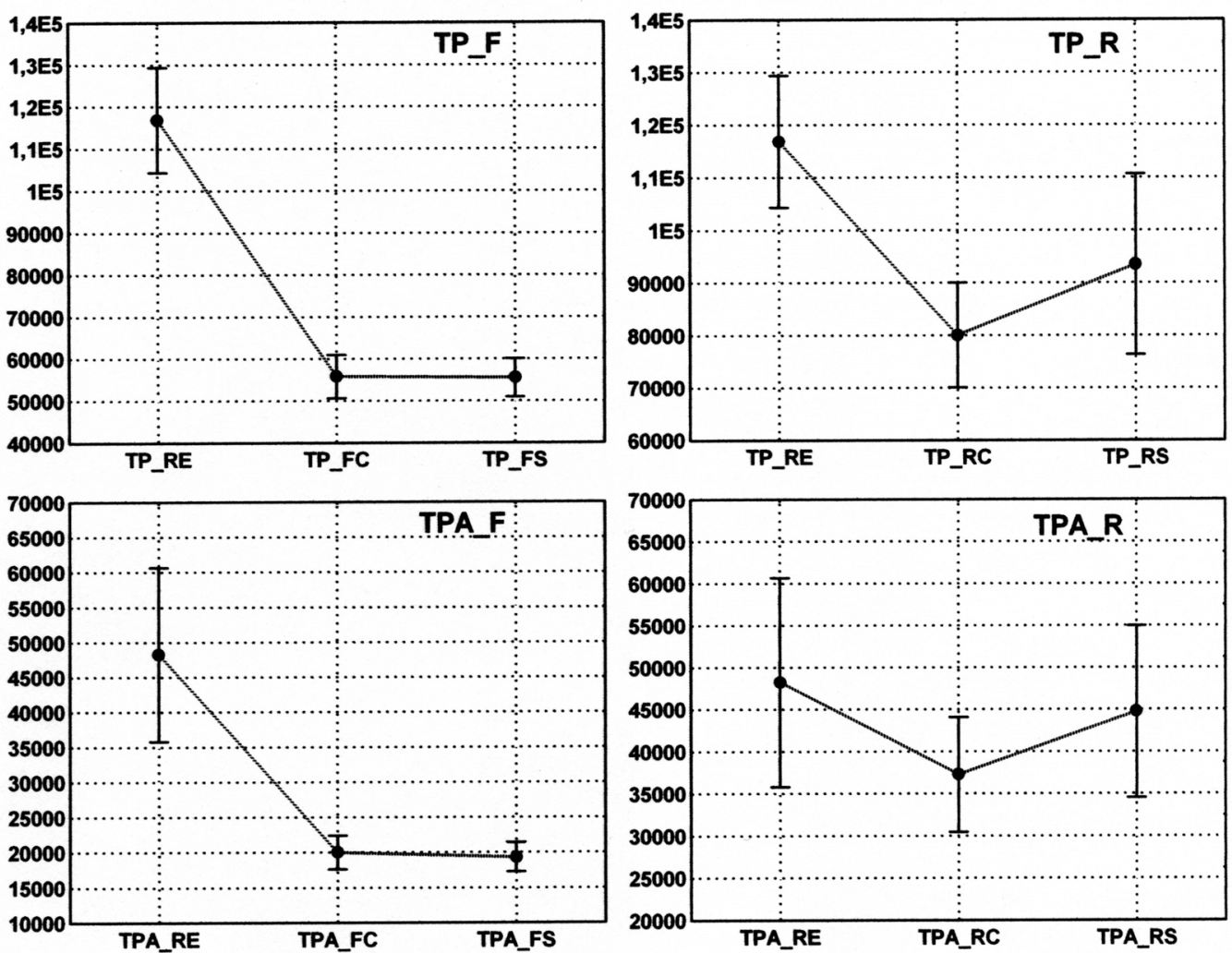

Fig. 1. Average Total Power values calculated for the 3 to $20 \mathrm{~Hz}$ (TP) and for the 8 to $13 \mathrm{~Hz}$ (TPA) EEG frequency bands during the cognitive tasks solving period (TP/TPA_F) and during the recovery period (TP/ TPA_R). Abscissa: TP/TPA_RE - presentation of reference stimuli; TP/TPA_FC(FS) - presentation of "unfolded cubes"/("nets of sixteen small squares") during the cognitive task solving period (left half,_F) and during the recovery period (right half, _R). Ordinate: TP in $\mathrm{mV}^{2}$. Black circles - average values, Vertical bars -0.95 per cent confidence intervals.

digitally filtered records for the Fast Fourier Transform analysis (FFT) - the starting points of the cut segments of EEG record were time-locked to the photodiode signal.

For each experimental task and everybody's subject the SciWork FFT Analysis module calculated the total power histograms from a fixed frequency band $(3-20 \mathrm{~Hz}$ or $8-13 \mathrm{~Hz}$ ) for each 3 -second EEG sample with $50 \%$ overlay and all electrodes. From the histograms the software derived two FFT single values - Total Power and Frequency at Maximum Power, averaged either of them separately and the actual average numerical values visualized in a spreadsheet format on the screen DataPad Plugin module. Subsequent statistical data analyses were accomplished with the StatSoft software package (StatSoft, Tulsa, OK).

The average decrease/increase in Total Power and Frequency at Maximum Power expressed as percentage of decrease in TP/FMP within the frequency band of interest in the period of the mental task solving as compared to the reference interval (passive viewing of a blank white oval) was calculated in each subject. Then the average TP(TPA)/FM(FMA) values were correlated with the score of FPI parameters of Freiburg Personality Inventory ${ }^{7}$ to ascertain their relations.

\section{RESULTS}

Total Power for the 3 to $20 \mathrm{~Hz}$ (TP) and for the 8 to $13 \mathrm{~Hz}$ (TPA) EEG frequency bands

Figs. 1 and 2 illustrate graphically the average energy of Total Power measured during the presentation of reference stimuli ${ }^{\mathrm{a}}$ and during the solution of the first ${ }^{\mathrm{b}}$ and the second ${ }^{c}$ cognitive tasks and in the rest period ${ }^{d}$.

From Fig. 1 (left column) it follows that during both cognitive tasks solving (_FC and _FS) the average values of TP_FC/FS as well as TPA_FC/FS were significantly lower than during the reference periods (TP/TPA_RE). However, the mean values calculated for the first (TP/ TPA_FC) and the second (TP/TPA_FS) cognitive tasks did not differ in magnitude.

On the other hand, in the rest period (the right column in Fig. 1) the dynamics of ongoing EEG was different. The first type of test visual stimuli (unfolded cube) induced more conspicuous ERD than did the second type of test visual stimuli (net of squares). The average TP/

\footnotetext{
a The period in which the white blank oval on a dark background appeared - reference stimuli.

b "Choose the cube that can be folded up from the unfolded cube!"

c "Replenish the missing part of a figure with the satisfactory item!"

d The period following successful mental task solution in which presentation of the first or second types of visual stimuli goes on.
} 

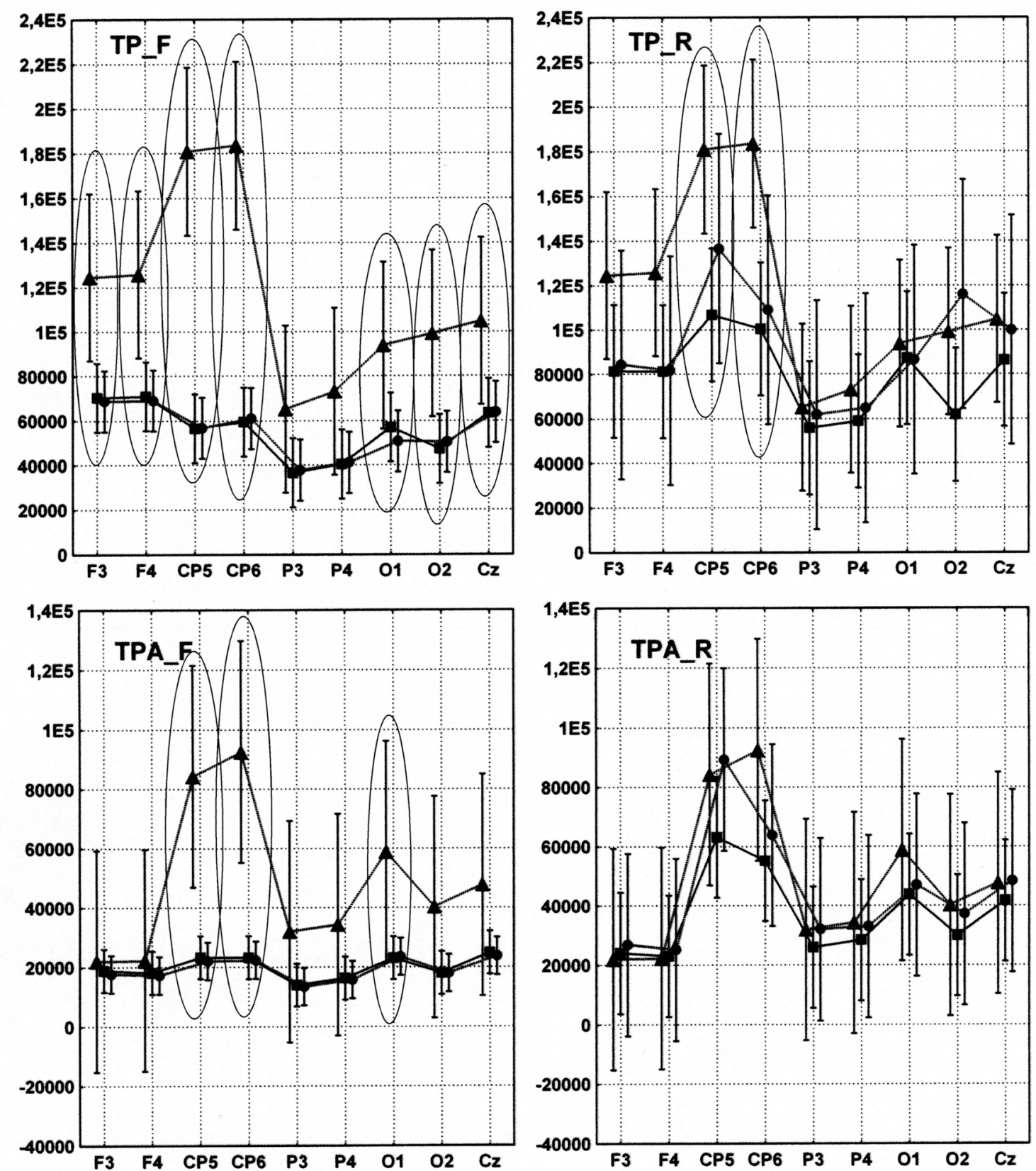

Fig. 2. Dependence of average Total Power values $\left[T P \_F(R) / T P A \_F(R)\right]$ on the site of recording electrode (Abscissa). The energy of TP/TPA during presentation of rest stimuli (triangles), unfolded cubes (squares) and nets of sixteen small squares (circles). Significant differences between averages $(p<0.05)$ are encircled. Black triangles, squares and circles - average values, Vertical bars - 0.95 per cent confidence intervals.

TPA values measured during the presentation of the first type of visual stimuli were significantly lower as values measured during the presentation of reference stimuli (blank white oval). The same dynamics showed also TP/ TPA values during action of the second type of test visual stimuli, nevertheless, in the alpha frequency band, the difference between mean TPA_RE and TPA_RS values was statistically insignificant (see bottom diagram in the right column in Fig. 1). Besides, it should be added here that differences between TP/TPA_RC vs. TP/TPA_RS were statistically significant.

The results of Repeated Measures ANOVA revealed significant interaction between the site of scalp recording electrode and the average TP/TPA value. They were espe- cially parietal electrodes CP5 and CP6, which at the reference interval reached significantly higher average TP/TPA values, as did the other electrodes (see Fig. 2 - triangles)

During the solving of both cognitive tasks the average TP/TPA values were lower in all recording sites (see the left column in Fig. 2), as compared with reference TP/ TPA values. Nevertheless, the significant decrease of TP was related to all electrodes except P3 and P4 while TPA decreases significantly only at electrodes CP5, CP6 and $\mathrm{O} 1$.

At the rest period (right column in Fig. 2) the dynamics of TP/TPA was much simpler, as compared with cognitive task solving period. The average values of both tested parameters showed no significant differences at all 

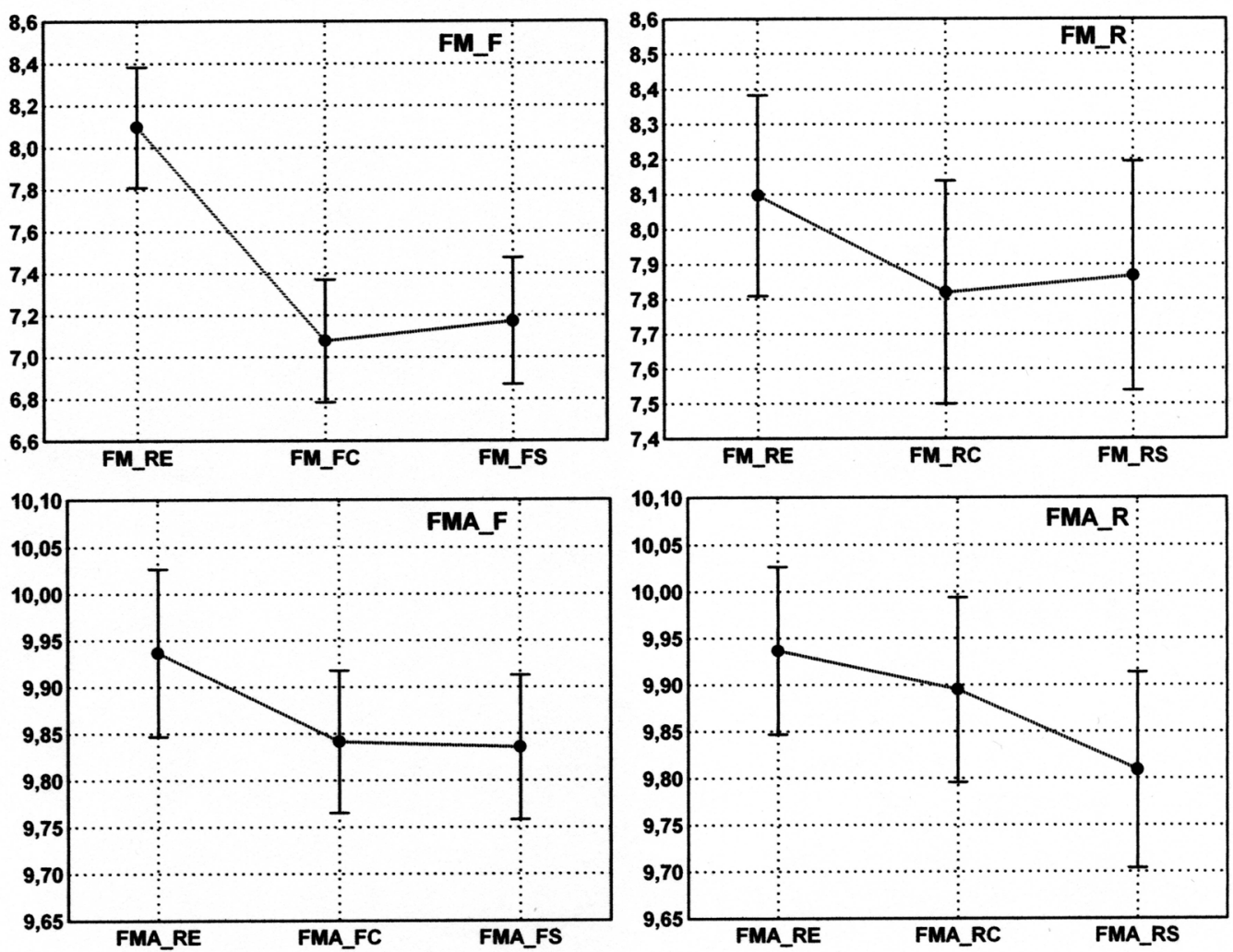

Fig. 3. Average Frequency at Maximum Power calculated for the 3 to $20 \mathrm{~Hz}$ (FM) and for the 8 to $13 \mathrm{~Hz}$ (FMA) EEG frequency bands during a cognitive tasks solving period (FM/FMA_F) and during a recovery period (FM/FMA_R).Abscissa: FM/FMA_RE - presentation of "reference stimuli"; FM/FMA_FC(FS) - presentation of "unfolded cubes"/("nets of sixteen small squares") during the cognitive task solving period (left half,_F) and during the recovery period (right half,_R). Ordinate: Frequency in Hz. Black circles - average values, Vertical bars - 0.95 per cent confidence intervals.

recording sites. The exception was the electrode $\mathrm{CP} 5$ and CP6, which showed significantly lower TP values during the presentation of both test stimuli than during the action of reference stimuli.

Frequency at Maximum Power for the 3 to $20 \mathrm{~Hz}$ (FM) and for the 8 to $13 \mathrm{~Hz}$ (FMA) EEG frequency bands

Figs. 3 and 4 demonstrate the dynamics of Frequency at Maximum Power (FM/FMA) in separate phases of our experiment. Inspection of Figures show that during the cognitive task solving period both FM and FMA showed similar changes as did TP and TPA - average FM/FMA frequencies significantly decreased during the cognitive task solving and the differences between the averages FM/ FMA_FC vs. FM/FMA_FS were insignificant, too.

On the other hand, in the rest period only FM but no FMA kept the described dynamics. The latter as compared to reference level was decreased only during the action of the second type of stimuli (net of squares). The stimuli of the first type (unfolded cube) produced no significant frequency change (see the bottom diagram in Fig. 3) - the average FMA_RC did not differed from FMA_RE.

The dependence of the average FM/FMA values on the site of recording electrode was revealed by detailed sta- tistical analysis. Fig. 4 illustrates this graphically. From it follows that regardless of the functional state of subject's brain (reference interval, rest phase, active mental task solving) average FM values were highest at occipital O1, $\mathrm{O} 2$ recording sites, lower at parietal CP5, CP6, P3, P4 and lowest at frontal F3, F4 sites. Average FMA values behaved similarly except for FMA at $\mathrm{O} 1$ and $\mathrm{O} 2$ recording sites - unlike $\mathrm{FM}$ at $\mathrm{O} 1$ and $\mathrm{O} 2$ the average FMA values did not differ from the average FMA measured at parietal electrodes.

The mental load related to solving each of the two cognitive tasks significantly decreased the average FM while it left the average FMA unchanged (compare diagrams in the left column in Fig. 4). No changes of the average FM/FMA values were observed in the rest phases during the presentation of whichever of three visual stimuli (reference stimulus and two test stimuli) - see the right column in Fig. 4.

For the sake of completeness, it should be added that none of the twelve personality traits assessed by Freiburg Personality Inventory (FPI) affected the size of event-related desynchronization/synchronization in our subjects - there were no significant correlations between the two variables. 

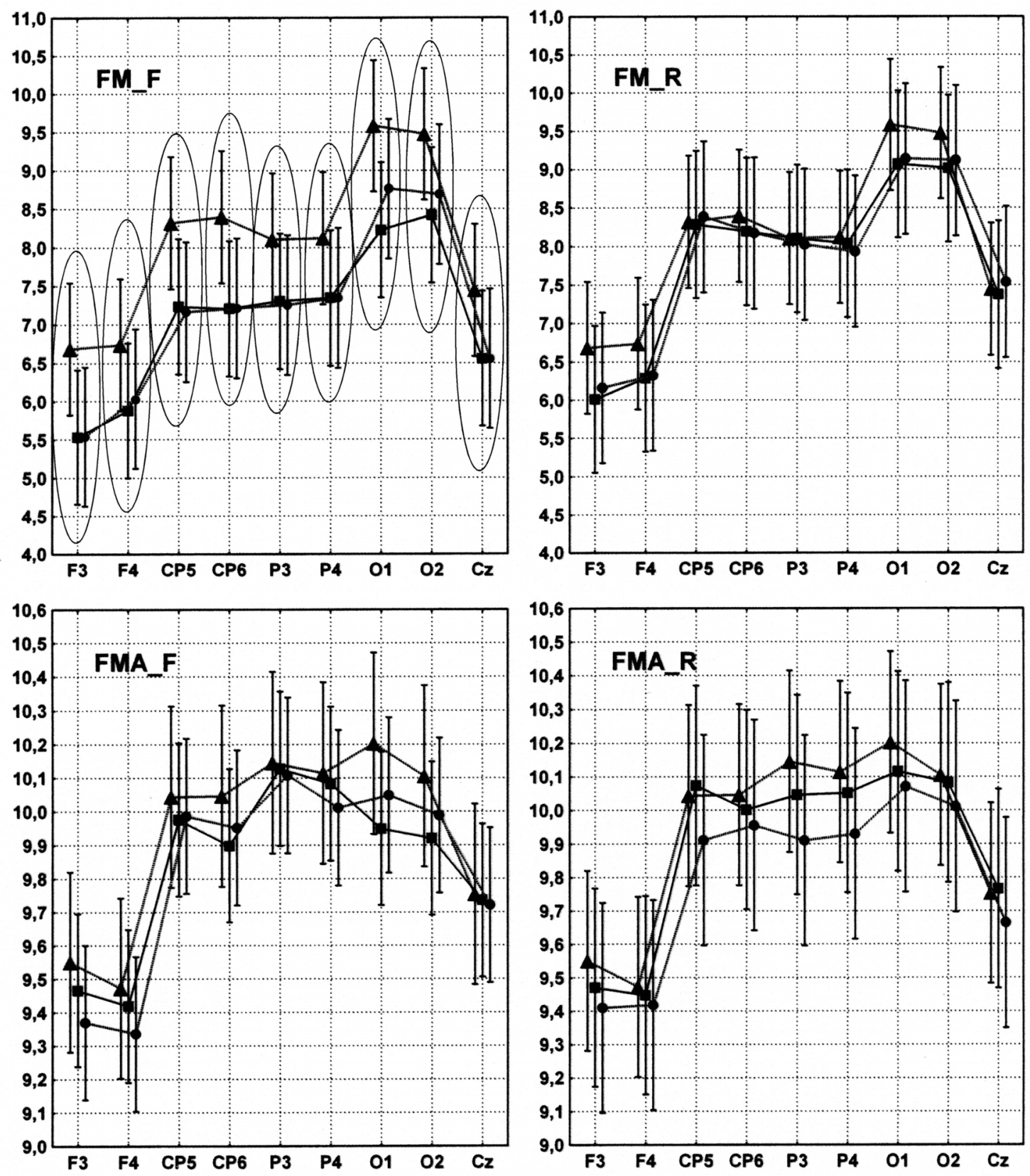

Fig. 4. Dependence of average values of Frequency at Maximum Power $\left[F M \_F(R) / F M A \_F(R)\right]$ on the site of recording electrode (Abscissa). Significant differences between averages $(p<0.05)$ are encircled. For other details see the text to Fig. 2 and 3.

\section{DISCUSSION}

The findings of our experiment demonstrate that during the solution of cognitive tasks marked changes in ongoing EEG activity appeared in all participants. The short lasting and localized amplitude decrease in rhythmic activity (ERD) and the change of EEG frequency were among the most frequent. It is not surprising because there is a number of literature data (see review of Klimesh $^{6}$ and monographs of Faber ${ }^{3,4}$ and Neuper and Klimesch ${ }^{13}$ ) demonstrating the relation of mental load to the event-related desynchronization/or synchronization. In accord with them are also our recent results ${ }^{14}$ showing that prominent ERD follows the higher mental load re- lated to active searching for identical pictures in a group of similar ones.

The results also revealed that the ERD extent is determined by an informational content of processed visual stimuli. All subjects showed very conspicuous ERD (average TP values - the quantitative measure of ERD extent - were smaller as the reference TP value) when the presented visual stimulus (unfolded cube or net of squares) ordered them to solve the first or the second prearranged cognitive tasks. When, however, it demanded no subject's cognitive activity (rest period of the experiment), ERD was less conspicuous.

The findings also suggest that not only the informational content of the stimulus but also the character of the 
stimulus per se can affect the ERD extent. In the rest period of our experiment, the presentation of unfolded cubes was followed by more marked ERD than the presentation of the net of squares - all of this occurred without any modification of original properties of both visual stimuli. Thus it seems that the extent of ERD reflects very well the momentary excitability of neural structures participating in successful processing of information in brain systems during the solving of a particular mental task.

The site of the scalp recording electrode represents another factor that determined the extent of ERD related to mental load in the course of different cognitive brain activities in the experiment. A higher mental load pertinent to the solved cognitive task resulted in prominent ERD in all recording sites except P3 and P4 electrodes.

The behaviour of the latter electrodes is somewhat surprising because parietal brain areas are certainly engaged in the processing of visual information related to mental task solving. This assumption is supported by our earlier findings ${ }^{14,15}$ as well as by the dynamics of average FM pertinent to cognitive task solving described in the present paper; the average FM calculated for each of EEG records including P3 and P4 significantly decreased during the mental load related to solving each of two cognitive tasks.

The finding that higher mental load related to the solution of cognitive tasks shifts average FM to lower frequencies is not surprising if we consider published

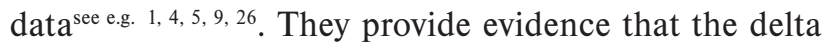
and sometimes theta activities increase in a direct, proportional way in all EEG records in the course of mental activity, this is, the greater the mental effort, the higher the delta activity.

\section{REFERENCES}

1. Bastiaansen MCM, Van Berkum JJA, Hagoort P. Event-related theta power increases in the human EEG during online sentence processing. Neurosci Letters 2002; 323:13-6.

2. Berger H. Úber das Electroencephalogramm des Menschen: 1. Mitteilung. Arch Psychiatr Nervenkr 1929; 87:527-8.

3. Faber J. Electroencephalography and psychophysiology (in Czech). Praha: ISV; 2000.

4. Faber J. QEEG: Correlation of EEG analysis with psychological tests. Praha: Galén; 2005.

5. Guderian S, Düzel E. Inducced theta oscillations mediate largescale synchrony with mediotemporal areas during recollection in humans. Hippocampus 2005; 15:901-12.

6. Klimesch W. EEG alpha and theta oscillation reflect cognitive and memory performance: a review and analysis. Brain Res Rev 1999; 29:169-95.
7. Kollarik T, Poliakova E, Ritomsky A. Freiburský osobnostný dotaznik (in Slovak). Bratislava: Psychodiagnostické a didaktické testy, 1984.

8. Krause CM, Lang AH, Laine M, Kuusisto M, Pörn B. Event-related desynchronization and synchronization during an auditory memory task. Electroenceph clin Neurophysiol 1996; 98:319-26.

9. Krause CM, Silanmäki L, Koivisto M, Saarela C, Häggqvist A, Laine M, Hämäläinen $\mathrm{H}$. The effects of memory load on eventrelated EEG desynchronization and synchronization. Clin Neurophysiol 2000; 111:2071-78.

10. Krause CM, Grönholm P, Leinonen A. Laine M, Säkkinen AL, Söderholm C. Modality matters: The effects of stimulus modality on the 4- to $30-\mathrm{Hz}$ electric oscillation during a lexical decision task. Brain Res 2006; 1110:182-192.

11. Mazaheri A, Picton TW. EEG spectral dynamics during discrimination of auditory and visual targets. Cogn Brain Res 2005; 24:81-96.

12. Morrel LK. Some characteristics of stimulus-provoked alpha activity. Electroenceph clin Neurophysiol 1996; 21:552-61.

13. Neuper C, Klimesch W, editors. Event-related dynamics of brain oscillations. Amsterdam: Elsevier; 2006.

14. Petrek J. Selection of two identical pictures from a group of similar ones: Changes in ongoing EEG activity. Activ Nerv Sup 2008; 50:3-8.

15. Petrek J. Pictorial cognitive task solving and dynamics of eventrelated potentials. Biomed Pap Med Fac Univ Palacky Olomouc Czech Repub 2008; 152:223-230.

16. Pesonen M, Björnberg CH, Hämäläinen H, Krause CM. Brain oscillatory 1-30 Hz EEG ERD/ERS responses during the different stages of an auditory memory search task. Neurosci Letters 2006; 399:45-50.

17. Pfurtscheller G. Graphical display and statistical evaluation of event-related desynchronization (ERD). Electroenceph clin Neurophysiol 1977; 43:757-60.

18. Pfurtscheller G. Event-related synchronization (ERS): an electrophysiological correlate of cortical areas at rest. Electroenceph clin Neurophysiol 1992; 83:62-9.

19. Pfurtscheller G.: Functional brain imaging based on ERD/ERS. Vision Res. 2001; 41:1257-60.

20. Pfurtscheller G. Induced oscillations in the alpha band: Functional meaning. Epilepsia 2003; 44 (Suppl 12):2-8

21. Pfurtscheller G, Aranibar A. Event-related cortical desynchronization detected by power measurements of scalp EEG. Electroenceph clin Neurophysiol 1977; 42:817-26.

22. Pfurtscheller G, Neuper C, Mohl W. Event-related desynchronization (ERD) during visual processing. Int J Psychophysiol 1994; $16: 147-53$.

23. Pfurtscheller G, Lopes da Silva FH. Event related EEG/MEG synchronization and desynchronization : basic principles. Clin Neurophysiol 1999; 110:1842-57.

24. Pfurtscheller G, Neuper C, Pichler-Zalaudek K, Edlinger G, Lopes da Silva FH. Do brain oscillations of different frequencies indicate interaction between cortical areas. Neurosci Letters 2000; 286:668.

25. Pulvermüller F, Birbaumer N, Lutzenberger W, Mohr B. Highfrequency brain activity: Its possible role in attention, perception and language processing. Progress Neurobiol 1997; 52:427-45.

26. Van Dijk JG, Caekebeke JFV, Jennekens-Schinkel A, Ywinderman AH. Background EEG activity in auditory event related potentials. Electroenceph clin Neurophysiol 1992; 83:44-51. 